\title{
A scheme to treat the singularity in global seismic wavefield simulation using pseudospectral method with staggered grids*
}

\author{
Yanbin Wang $^{1, *}$ and Hiroshi Takenaka ${ }^{2}$ \\ ${ }^{1}$ Department of Geophysics, School of Earth and Space Sciences, Peking University, Beijing 100871, China \\ ${ }^{2}$ Department of Earth and Planetary Sciences, Kyushu University, Hakozaki 6-10-1, Fukuoka 812-8581, Japan
}

\begin{abstract}
The pseudospectral method has been applied to the simulation of seismic wave propagation in 2-D global Earth model. When a whole Earth model is considered, the center of the Earth is included in the model and then singularity arises at the center of the Earth where $r=0$ since the $1 / r$ term appears in the wave equations. In this paper, we extended the global seismic wavefield simulation algorithm for regular grid mesh to staggered grid configuration and developed a scheme to solve the numerical problems associated with the above singularity for a 2-D global Earth model defined on staggered grid using pseudospectral method. This scheme uses a coordinate transformation at the center of the model, in which the field variables at the center are calculated in Cartesian coordinates from the values on the grids around the center. It allows wave propagation through the center and hence the wavefield at the center can be stably calculated. Validity and accuracy of the scheme was tested by compared with the discrete wavenumber method. This scheme could also be suitable for other numerical methods or models parameterized in cylindrical or spherical coordinates when singularity arises at the center of the model.
\end{abstract}

Key words: seismic modeling; wave propagation; whole Earth; pseudospectral method; staggered grid CLC number: P315.01 Document code: A

\section{Introduction}

Modeling seismic wavefield in global Earth model is important in understanding the physical process of elastic wave propagation through heterogeneous whole Earth. It provides potential forward modeling tools for waveform inversion to study global structure of the Earth's interior. With the development of computational performance, global seismic wavefield simulation has been performed using several numerical techniques.

Igel and Weber $(1995,1996)$ applied finite difference method (FDM) to simulate SH and P-SV wave propagation in an axisymmetric Earth model. Igel and Gudmundsson (1997) used FDM to study frequency dependent effects of long period S and SS waves. Jahnke et al (2008) presented a parallel FDM scheme for global SH wave simulation and modeled the effect of whole

\footnotetext{
* Received 8 January 2010; accepted in revised form 4 February 2010; published 10 April 2010.

^ Corresponding author. e-mail: ybwang@pku.edu.cn

(C) The Seismological Society of China and Springer-Verlag Berlin Heidelberg 2010
}

mantle scattering. Toyokuni et al (2005) developed a quasi-spherical approach based on FDM for 2-D slice of global model. Komatitsch and Tromp (2002) and Nissen-Meyer et al (2008) applied the spectral element method (SEM) to 3-D global model and a spherical symmetric Earth model, respectively. Yan et al (2009) modeled regional wave propagation caused by the Wenchuan earthquake using SEM. Cummins et al (1994a, b) developed a direct solution method for spherical symmetric whole Earth model.

Furumura et al (1998) developed a pseudospectral method (PSM) for 2-D whole Earth model defined in cylindrical coordinate. This method effectively modeled global P-SV wavefield down to a depth of $5000 \mathrm{~km}$. However, the center of the Earth could not be included into their model due to the singularity of wave equations at the Earth's center $(r=0)$. Wang et al (2001) successfully solved the numerical problem associated with the singularity and presented a PSM scheme for 2-D whole Earth P-SV wave propagation. Both of the above two PSM schemes discretize the model using regular grid 
mesh, in which all the filed variables and medium parameters are defined at the same spatial location. This could cause numerical errors at the discontinuities in the model, such as the liquid-solid inner core boundary and the free surface. A staggered grid PSM is efficient to reduce such errors and improve the accuracy of the simulation compared to the regular grids (Özdenvar and McMechan, 1996).

In this paper, we extended the PSM method (Wang et al, 2001) to a staggered grid mesh for 2-D whole Earth model defined in cylindrical coordinate. We developed a new scheme to solve the singularity problem associated with the center of the Earth for staggered grid configuration. The validity and accuracy of the scheme was tested with a simple model problem by comparing with the discrete wavenumber method.

\section{Wave equations and staggered gird mesh}

We consider a whole Earth model defined in 2-D cylindrical coordinates $(r, \theta)$ with $0 \leq r \leq \infty$ and $0 \leq \theta \leq 2 \pi$, and the corresponding wave equations for P-SV motion in velocity-stress form are given by

$$
\left\{\begin{array}{l}
\rho \frac{\partial v_{r}}{\partial t}=\frac{1}{r} \frac{\partial}{\partial r}\left(r \sigma_{r r}\right)+\frac{1}{r} \frac{\partial \sigma_{r \theta}}{\partial \theta}-\frac{\sigma_{\theta \theta}}{r}+f_{r} \\
\rho \frac{\partial v_{\theta}}{\partial t}=\frac{1}{r^{2}} \frac{\partial}{\partial r}\left(r^{2} \sigma_{r \theta}\right)+\frac{1}{r} \frac{\partial \sigma_{\theta \theta}}{\partial \theta}+f_{\theta}
\end{array},\right.
$$

where $v_{r}$ and $v_{\theta}$ are the velocity components, $\rho$ is mass density, $f_{r}$ and $f_{\theta}$ are body forces, and $\sigma_{r r}, \sigma_{r \theta}, \sigma_{\theta \theta}$ are stress components. The constitutive relations between the stress and velocity for an isotropic linear elastic solid are

$$
\left(\begin{array}{l}
\frac{\partial \sigma_{r r}}{\partial t}=(\lambda+2 \mu) \frac{\partial v_{r}}{\partial r}+\frac{\lambda}{r}\left(\frac{\partial v_{\theta}}{\partial \theta}+v_{r}\right) \\
\frac{\partial \sigma_{\theta \theta}}{\partial t}=\lambda \frac{\partial v_{r}}{\partial r}+\frac{\lambda+2 \mu}{r}\left(\frac{\partial v_{\theta}}{\partial \theta}+v_{r}\right) \\
\frac{\partial \sigma_{r \theta}}{\partial t}=\frac{\mu}{r}\left(\frac{\partial v_{r}}{\partial \theta}-v_{\theta}\right)+\mu \frac{\partial v_{\theta}}{\partial r}
\end{array}\right.
$$

where $\lambda$ and $\mu$ are the Lame constants.

To solve the above equations for a 2-D whole Earth model, we discretize the whole physical domain in the radial $(r)$ and lateral $(\theta)$ directions in 2-D cylindrical coordinates using staggered grids as shown in Figure 1. In pseudospectral method with staggered grids, the spatial derivatives with respect to $r$ and $\theta$ in above equations are calculated half-way between grid points $(m \pm 1 / 2) \Delta x$ via fast Fourier transformation by (Zhao et al, 2001)

$$
\begin{gathered}
\frac{\mathrm{d}}{\mathrm{d} x} f\left[\left(m \pm \frac{1}{2}\right) \Delta x\right]= \\
\frac{\Delta k}{2 \pi} \sum_{l=0}^{N-1} \mathrm{i}(l \Delta k) \mathrm{e}^{ \pm \mathrm{i} l \Delta k \Delta x / 2} F(l \Delta k) \mathrm{e}^{\mathrm{i} 2 \pi l m / N} \\
m=0,1, \cdots, N-1,
\end{gathered}
$$

where $f(\Delta x)$ represents the discretized field variables in the wave equations, $F(l \Delta k)$ is the Fourier transformation of $f(\Delta x)$ in wavenumber domain, $N$ is the number of grid points. The time domain differentiation in wave equations is performed by a finite difference scheme as used in Wang et al (2001).

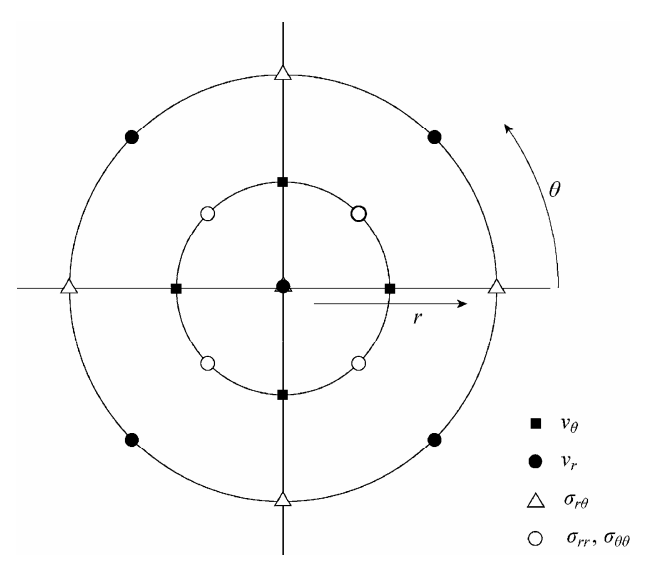

Figure 1 Configuration of the grid distribution on staggered grids for the 2-D whole Earth model including the center. $v_{r}$ and $\sigma_{r \theta}$ are located at the center.

\section{Solve the singularity at the center}

Wang et al (2001) considered how to avoid the singularity at the center of the above 2-D model when the numerical mesh is defined on regular grids. In their method, the singularity problem is solved by avoiding the calculation at the center using an extension scheme and the grid points are located around the center. But in staggered grids, as shown in Figure 1, the stress and velocity components are located at different grid points. Therefore some field variables have to be defined on the grid point at the center.

In this section, we describe how to treat the singularity at center of the model on staggered grids when some of the field variables are defined at center and the wave equations have to be solved on the grid at center. As shown in Figure 1, the radial velocity component $v_{r}$ and stress component $\sigma_{r \theta}$ are defined on the surface and 
then these two variables have to be defined on the center As same as in Wang et al (2001), the calculation of the spatial derivatives in this paper are performed on $-R \leq r \leq+R$ ( $R$ is the radius of the Earth) and $0 \leq \theta \leq 2 \pi$ if the center is included, the values of $v_{r}$ and $\sigma_{r} \theta$ at the center are used in the operations to calculate the spatial derivatives. But all the grid points in $\theta$ direction fall into only one grid point at the center and the values of $v_{r}$ and $\sigma_{r \theta}$ should be fixed but not vary with $\theta$.

To calculate the values of $v_{r}$ and $\sigma_{r \theta}$ at the center, we propose a transformation of coordinate systems around the center, and these values will be calculated from the values of corresponding velocity and stress components at or around the center in Cartesian coordinates. As defined in Figure 2, the $x$ and $z$ axes in Cartesian coordinates are located at $\theta=0$ and $\theta=\pi / 2$, respectively. The velocity component $v_{r}$ and $v_{\theta}$ in cylindrical coordinates are related to $v_{x}$ and $v_{z}$ in Cartesian coordinates through the transformation matrix in the following equation:

$$
\left(\begin{array}{c}
v_{r} \\
v_{\theta}
\end{array}\right)=\left(\begin{array}{cc}
\cos \theta & \sin \theta \\
-\sin \theta & \cos \theta
\end{array}\right)\left(\begin{array}{l}
v_{x} \\
v_{z}
\end{array}\right)
$$

Therefore, $v_{r}$ and $v_{\theta}$ at the center can be calculated from $v_{x}$ and $v_{z}$ at this grid point.

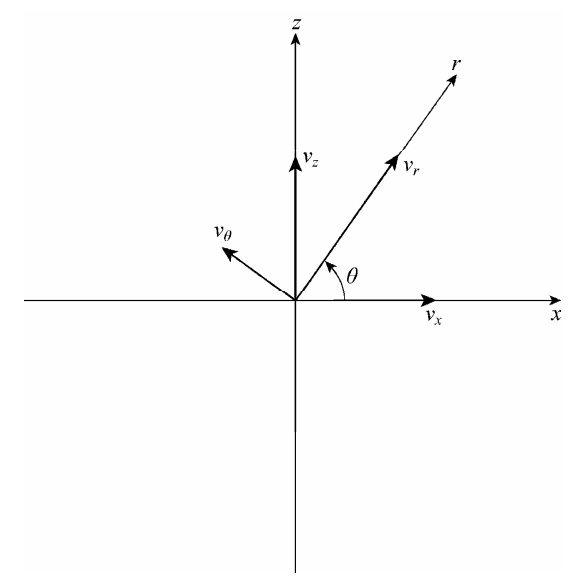

Figure 2 Conversion between $v_{r}$ and $v_{\theta}$ in cylindrical coordinates and $v_{x}$ and $v_{z}$ in Cartesian coordinates at the center of the model. The origin of the Cartesian coordinates is the center of the model.

The values of $v_{x}$ and $v_{z}$ at the center can be calculated from the $v_{\theta}$ values around the center. We observe the velocity components on grid points around the center as shown in Figure 3a; the distance of these four grid points to the center are $\Delta r / 2$ in cylindrical coordinates and the corresponding distances to the center in Cartesian coordinates are $\Delta x / 2$ and $\Delta z / 2$, where $\Delta x=\Delta r$ and $\Delta z=\Delta r$. Therefore, the relationship between the values of $v_{x}$ and $v_{\theta}$ on the grid points in Figure $3 \mathrm{a}$ is

$$
\left\{\begin{array}{l}
v_{x}(x=0, z=-\Delta z / 2)=v_{\theta}(r=\Delta r / 2, \theta=3 \pi / 2) \\
v_{x}(x=0, z=\Delta z / 2)=-v_{\theta}(r=\Delta r / 2, \theta=\pi / 2)
\end{array} .\right.
$$

At the center $v_{x}$ is equal to $v_{\theta}$, which can be calculated by a shift of $\Delta r / 2$ along $z$ axis for $v_{\theta}$ on all grid points at $\theta=\pi / 2$ and $\theta=3 \pi / 2$ performed in wavenumber domain via fast Fourier transformation as used in Wang et al (2001).
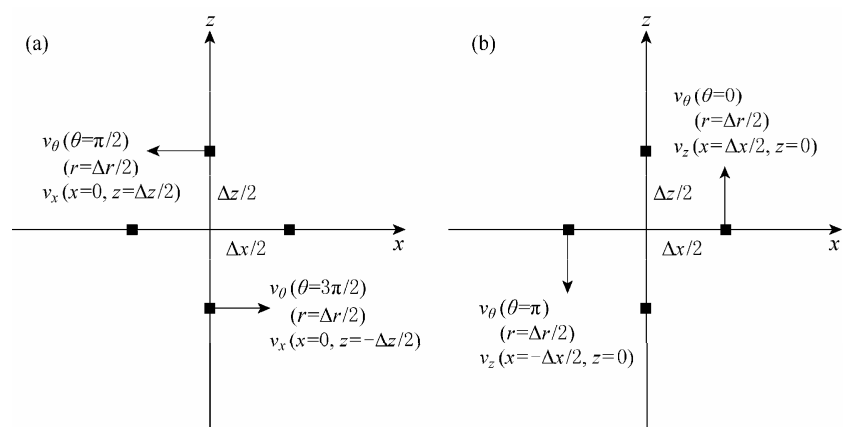

Figure 3 Relationship between $v_{\theta}$ in cylindrical coordinates and $v_{x}(\mathrm{a}), v_{z}(\mathrm{~b})$ in Cartesian coordinates. Filled squares are the location of $v_{\theta}$ as shown in Figure 1.

The relationship between values of $v_{z}$ and $v_{\theta}$ on the grid points, as shown in Figure 3b, are given by

$$
\left\{\begin{array}{l}
v_{z}(x=\Delta x / 2, z=0)=v_{\theta}(r=\Delta r / 2, \theta=0) \\
v_{z}(x=-\Delta x / 2, z=0)=-v_{\theta}(r=\Delta r / 2, \theta=\pi)
\end{array} .\right.
$$

At the center $v_{z}$ equals to $v_{\theta}$, which can be calculated by a shift of $\Delta r / 2$ along the $x$ axis for $v_{\theta}$ on all grid points at $\theta=0$ and $\theta=\pi$ performed in wavenumber domain as well.

After we calculated the values of $v_{x}$ and $v_{z}$ at the center $(x=0$ and $z=0)$ in Cartesian coordinates from the $v_{\theta}$ values, $v_{r}$ at the center can be calculated from the relations in equation (4) by

$$
\begin{gathered}
v_{r}(r=0)=\cos \theta \cdot v_{x}(x=0, z=0)+ \\
\sin \theta \cdot v_{z}(x=0, z=0) .
\end{gathered}
$$

Next, we show how to calculate $\sigma_{r \theta}$ at the center. The relationship between the stress components in 2-D cylindrical coordinates and 2-D Cartesian coordinates can be expressed by using the transformation matrix between coordinates similar to equation (4) as 


$$
\begin{gathered}
\left(\begin{array}{ll}
\sigma_{r r} & \sigma_{r \theta} \\
\sigma_{\theta r} & \sigma_{\theta \theta}
\end{array}\right)=\left(\begin{array}{cc}
\cos \theta & \sin \theta \\
-\sin \theta & \cos \theta
\end{array}\right)\left(\begin{array}{ll}
\sigma_{x x} & \sigma_{x z} \\
\sigma_{z x} & \sigma_{z z}
\end{array}\right)\left(\begin{array}{cc}
\cos \theta & -\sin \theta \\
\sin \theta & \cos \theta
\end{array}\right)= \\
\left(\begin{array}{cc}
\cos \theta \sigma_{x x}+\sin \theta \sigma_{z x} & \cos \theta \sigma_{x z}+\sin \theta \sigma_{z z} \\
-\sin \theta \sigma_{x x}+\cos \theta \sigma_{z x} & -\sin \theta \sigma_{x z}+\cos \theta \sigma_{z z}
\end{array}\right) . \\
\left(\begin{array}{cc}
\cos \theta & -\sin \theta \\
\sin \theta & \cos \theta
\end{array}\right) .
\end{gathered}
$$

Then, $\sigma_{r \theta}$ at the center for any $\theta$ can be calculated from $\sigma_{x x}, \sigma_{x z}$ and $\sigma_{z z}$ at the center by

$$
\sigma_{r \theta}(r=0)=-\frac{1}{2}\left(\sigma_{x x}-\sigma_{z z}\right) \sin 2 \theta+\sigma_{x z} \cos 2 \theta
$$

Therefore, we have to calculate $\sigma_{x x}, \sigma_{x z}$ and $\sigma_{z z}$ at the center in order to get the value of $\sigma_{r \theta}$ at the center.

As given in the constitutive relations in Cartesian coordinates $(x, z)$ in Wang et al (2001), the stress components $\sigma_{x x}, \sigma_{x z}$ and $\sigma_{z z}$ are calculated from the spatial derivatives $\partial v_{x} / \partial x, \partial v_{x} / \partial z, \partial v_{z} / \partial x$ and $\partial v_{z} / \partial z$. These spatial derivatives have to be evaluated in order to calculate the stress components in Cartesian coordinates. According to the relations given in equation (5) and diagrammatically shown in Figure $3 \mathrm{a}, \partial v_{x} / \partial z$ is equivalent to $\partial v_{\theta} / \partial r$ at $\theta=3 \pi / 2$ and $\theta=\pi / 2\left(\partial v_{r} / \partial z=\partial v_{\theta} / \partial r\right) . \partial v_{\theta} / \partial r$ at the center can be calculated from $v_{\theta}$ on all grid points along $\theta=3 \pi / 2$ and $\theta=\pi / 2$ by a shift of $\Delta r / 2$ along the $z$ axis. The calculated $\partial v_{\theta} / \partial r$ at the center is then the value of $\partial v_{x} / \partial z$ at the center. Similarly, the relations given in equation (6) and Figure $3 \mathrm{~b}$ allow $\partial v_{z} / \partial x$ to be calculated by the spatial derivatives on staggered grids for the $v_{\theta}$ on all grid points at $\theta=0$ and $\theta=\pi$ along the $x$ axis since $\partial v_{z} / \partial x=\partial v_{\theta} / \partial r$ holds at the center.

The calculations of $\partial v_{x} / \partial x$ and $\partial v_{z} / \partial z$ at the center are performed by evaluating $\partial v_{r} / \partial r$ at $\theta=\Delta \theta / 2$ and $\theta=\pi+$ $\Delta \theta / 2$. In Figure $4 \mathrm{a}, v_{r}$ at $\theta=\Delta \theta / 2$ and $\theta=\pi+\Delta \theta / 2$ are located on the $x^{\prime}$ axis in the Cartesian coordinates $\left(x^{\prime}, z^{\prime}\right)$, which is $\Delta \theta / 2$ rotated from the Cartesian coordinates $(x, z) . v_{x^{\prime}}$ in $\left(x^{\prime}, z^{\prime}\right)$ is equivalent to $v_{r}$. Since $v_{r}$ around the center are $\Delta r$ apart from the center and $v_{r}$ at the center can be calculated by above mentioned scheme [equation (7)], $\partial v_{r} / \partial r$ on all grid points along the $x^{\prime}$ axis at $\theta=\Delta \theta / 2$ and $\theta=\pi+\Delta \theta / 2$ can be calculated by the spatial differential operation on regular grids. The $\partial v_{x^{\prime}} / \partial x^{\prime}$ can then be calculated from the $\partial v_{r} / \partial r$ by $\partial v_{x^{\prime}} / \partial x^{\prime}=\partial v_{r} / \partial r$.

Similarly in Figure $4 \mathrm{~b}, \partial v_{r} / \partial r$ are calculated for all grid points along the $z^{\prime}$ axis at $\theta=\pi / 2+\Delta \theta / 2$ and $\theta=3 \pi / 2+$ $\Delta \theta / 2$ by spatial differential operation on regular grids and then $\partial v_{z^{\prime}} / \partial z^{\prime}$ at the center are calculated from $\partial v_{r} / \partial r$ at the center by $\partial v_{z^{\prime}} / \partial z^{\prime}=\partial v_{r} / \partial r$.

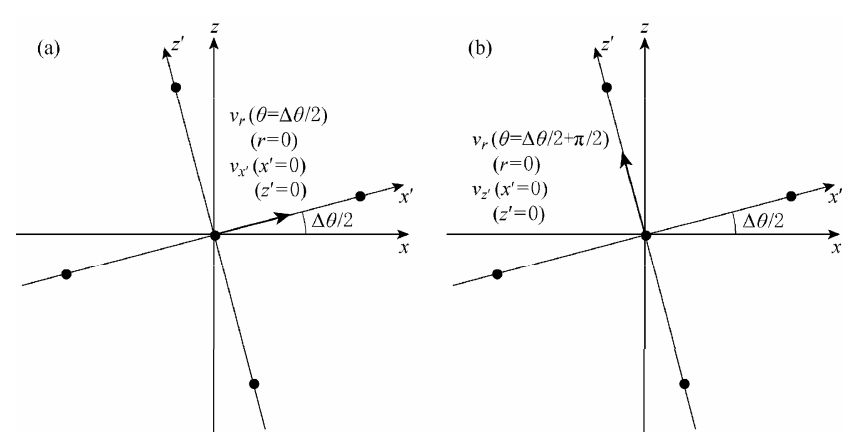

Figure 4 Relationship between $v_{r}$ in cylindrical coordinates and $v_{x^{\prime}}(\mathrm{a}), v_{z^{\prime}}(\mathrm{b})$ in Cartesian coordinates. Solid circles are the location of $v_{r}$ as shown in Figure 1. $v_{r}$ at $r=0$ is calculated by the above scheme given in equation (7).

$\sigma_{x^{\prime} x^{\prime}}$ and $\sigma_{z^{\prime} z^{\prime}}$ in coordinates $\left(x^{\prime}, z^{\prime}\right)$ can be calculated in Cartesian coordinates using constitutive relations given in Wang et al (2001). The stress components in $(x, z)$ and $\left(x^{\prime}, z^{\prime}\right)$ hold the following relations from the rotation of $\Delta \theta / 2$ between the two Cartesian coordinates:

$$
\left\{\begin{array}{l}
\sigma_{x^{\prime} x^{\prime}}=\cos ^{2}\left(\frac{\Delta \theta}{2}\right) \sigma_{x x}+\sin ^{2}\left(\frac{\Delta \theta}{2}\right) \sigma_{z z}+\sin (\Delta \theta) \sigma_{x z} \\
\sigma_{z^{\prime} z^{\prime}}=\sin ^{2}\left(\frac{\Delta \theta}{2}\right) \sigma_{x x}+\cos ^{2}\left(\frac{\Delta \theta}{2}\right) \sigma_{z z}-\sin (\Delta \theta) \sigma_{x z}
\end{array}\right.
$$

Hence $\sigma_{x x}$ and $\sigma_{z z}$ at the center in $(x, z)$ can be calculated by

$$
\left\{\begin{array}{c}
\sigma_{x x}=\frac{\cos ^{2}\left(\frac{\Delta \theta}{2}\right) \sigma_{x^{\prime} x^{\prime}}-\sin ^{2}\left(\frac{\Delta \theta}{2}\right) \sigma_{z^{\prime} z^{\prime}}-\sin (\Delta \theta) \sigma_{x z}}{\cos ^{4}\left(\frac{\Delta \theta}{2}\right)-\sin ^{4}\left(\frac{\Delta \theta}{2}\right)} \\
\sigma_{z z}=\frac{\sin ^{2}\left(\frac{\Delta \theta}{2}\right) \sigma_{x^{\prime} x^{\prime}}-\cos ^{2}\left(\frac{\Delta \theta}{2}\right) \sigma_{z^{\prime} z^{\prime}}-\sin (\Delta \theta) \sigma_{x z}}{\sin ^{4}\left(\frac{\Delta \theta}{2}\right)-\cos ^{4}\left(\frac{\Delta \theta}{2}\right)},
\end{array},\right.
$$

where $\sigma_{x z}$ can be directly calculated from $\partial v_{z} / \partial z$ and $\partial v_{z} / \partial x$ using the constitutive relation in Cartesian coordinates $(x, z)$. After we get the values of $\sigma_{x x}, \sigma_{x z}$ and $\sigma_{z z}$ at the center, $\sigma_{r} \theta$ at the center can be calculated by equation (9). Equation (11) may also be simply derived by using inverse transform in equation (8).

In the 2-D whole Earth model including the center defined on staggered grids, the spatial derivative calculations are performed on $-R \leq r \leq R$ instead of $0 \leq r \leq R$, 
which is similar to the treatment in the model defined on regular grids as in Wang et al (2001). In the radial (r) direction, a multi-domain scheme is used to enlarge the mesh size around the center of the Earth (Wang and Takenaka, 2001). Therefore, most of the operations mentioned in the above procedure to calculate $v_{r}$ and $\sigma_{r \theta}$ are already performed in the evaluation of spatial derivatives, there will be only a few extra operations needed to implement the above calculations. In each time step, the above scheme is accomplished by the following procedures:

1) Calculate $v_{x}$ and $v_{z}$ from $v_{\theta}$ at the center using equations (5) and (6);

2) Calculate $v_{r}$ at the center for all $\theta$ from $v_{x}$ and $v_{z}$ using equation (7);

3) Calculate $\partial v_{x} / \partial z, \partial v_{z} / \partial x$ and then $\sigma_{x z}$ at the center from $\partial v_{\theta} / \partial r$

4) Calculate $\partial v_{x^{\prime}} / \partial x^{\prime}, \partial v_{z^{\prime}} / \partial z^{\prime}$ and then $\sigma_{x^{\prime} x^{\prime}}$ and $\sigma_{z^{\prime} z^{\prime}}$ in Cartesian coordinates $\left(x^{\prime}, z^{\prime}\right)$ from $\partial v_{r} / \partial r$;

5) Calculate $\sigma_{x x}$ and $\sigma_{z z}$ from $\sigma_{x^{\prime} x^{\prime}}, \sigma_{z^{\prime} z^{\prime}}$ and $\sigma_{x z}$ using equation (11);

6) Calculate $\sigma_{r \theta}$ at the center from $\sigma_{x x}, \sigma_{x z}$ and $\sigma_{z z}$.

\section{Numerical tests of the accuracy}

We tested the accuracy of the scheme for evaluating the field variables at the center on staggered grids by comparing the results calculated using the present method and those obtained by using the discrete wavenumber method for P-SV wave propagation. The configuration of the grid distribution is the same as shown in Figure 1, $v_{r}$ and $\sigma_{r \theta}$ are located at the center. The model is a homogeneous disc defined in 2-D cylindrical coordinates $(r, \theta)$ with a radius of $6371.0 \mathrm{~km}$, $v_{\mathrm{P}}=10.0 \mathrm{~km} / \mathrm{s}, v_{\mathrm{S}}=7.0 \mathrm{~km} / \mathrm{s}$ and $\rho=3.3 \mathrm{~g} / \mathrm{cm}^{3}$. The source is a double-couple point source with moment tensor component $M_{r r}=-1.0 \times 10^{12} \mathrm{~N} \cdot \mathrm{m}, M_{\theta \theta}=1.0 \times 10^{12} \mathrm{~N} \cdot \mathrm{m}$, which is located at a depth of $3000 \mathrm{~km}$. The source time function and the spatial distribution of the body force are approximated by a pseudo-delta function (Herrmann, 1979). The width of the source time function is $60.0 \mathrm{~s}$. The numbers of grid points are 512 in the lateral direction and 128 in the radial direction. Smoothing in the wavenumber domain is performed by filtering out the high wavenumber components when calculating lateral derivatives, which is applied at the area near to the center to increase the time interval (Wang et al, 2001). The locations of the source and the five observation points are shown in Figure 5a.
The comparison calculations are performed by using the discrete wavenumber method (Bouchon and Aki, 1977) for a half-space model as displayed in Figure 5b. The relative locations between the source and the five observation points are the same in the half-space model as in the homogeneous disc model.

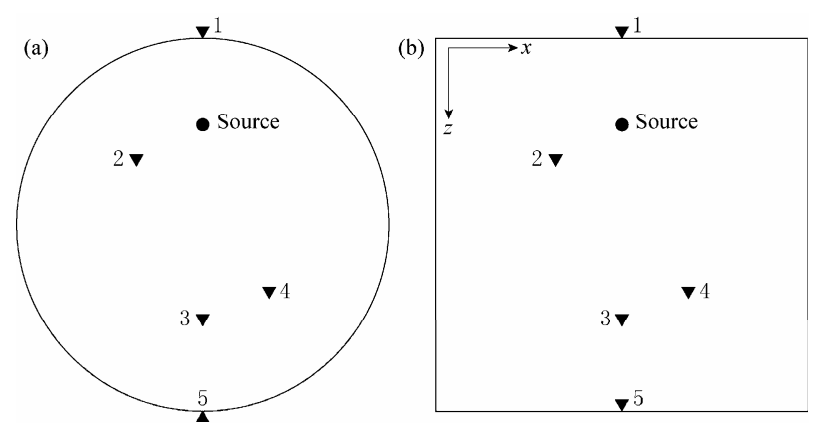

Figure 5 The model used for the comparison calculations to check the accuracy of the above discussed schemes to evaluate the values of field variables located at the center on staggered grids. (a) Homogeneous disc model in 2-D cylindrical coordinates $(r, \theta)$ with radius of $6371.0 \mathrm{~km}$; (b) homogeneous half-space model used for the discrete wavenumber calculation. Circle is the source and triangles are the observation points. The relative locations between the source and the observation points are the same in (a) and (b).

Figure 6 shows the synthetic seismograms for the comparing calculations. Since only the direct $\mathrm{P}$ and $\mathrm{S}$ waves are comparable between the two models, only these two direct arrivals are displayed. From these figures, we see very good agreement of both arrival times and amplitudes for $\mathrm{P}$ and $\mathrm{S}$ waves, which suggests that the proposed scheme to evaluate the field variables at the center is valid and accurate.

\section{Discussion and conclusions}

We have presented a scheme for solving numerical problems associated with singularity caused by the center of the Earth for 2-D global wavefield simulation using staggered grid mesh. This scheme uses coordinate transformation to evaluate filed variables at the center, which is necessary in spatial derivative calculations. Implementation of this scheme is straight forward and only needs a few operations. However, it extends the global wavefield simulation method of Wang et al (2001) from regular grid to staggered grid configuration, which could reduce the errors caused by the liquid-solid inner core boundary and free surface boundary. Although it is developed for 2-D cylindrical whole Earth model using 

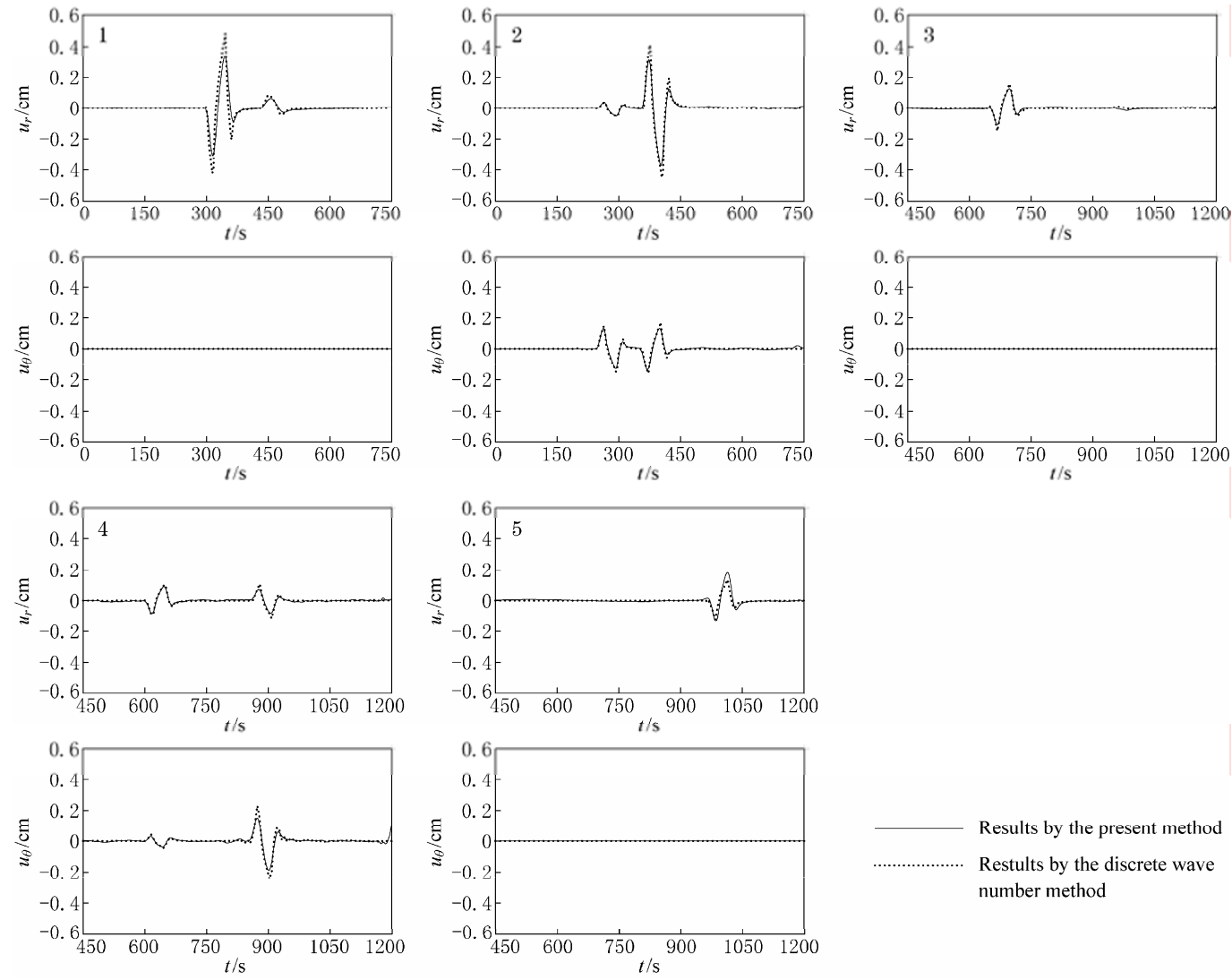

Figure 6 Synthetic seismograms of displacement at five observation points. The solid and dotted lines are the results by the present method and the discrete wavenumber method, respectively.

pseudospectral method, the scheme itself is independent of the specific numerical method used and the model involved. For example, it could be applied to global seismic wave propagation using staggered grid FDM or 3-D global model defined in spherical coordinate when the center of the Earth is included and the values of field variables at the center are needed in the calculations. It could also be applied to other geophysical problems if the model is defined in 2-D cylindrical or 3-D spherical coordinates including the center.

Comparison with the results of discrete wavenumber method showed validity and good accuracy of the scheme. In the future work, we will apply it to a heterogeneous 2-D cylindrical whole Earth model to study heterogeneous effect on global seismic wave propagation.

Acknowledgements This work was supported by the National Natural Science Foundation of China under grant Nos. 40474012,40874020 and 40821062.

\section{References}

Bouchon M and Aki K (1977). Discrete wave-number representation of seismic source wave fields. Bull Seis Soc Amer 67: 259-277.

Cummins P R, Geller R J, Hatori T and Takeuchi N (1994a). DSM complete synthetic seismograms: SH, spherically symmetric case. Geophys Res Lett 21: $533-536$.

Cummins P R, Geller R J and Takeuchi N (1994b). DSM complete synthetic seismograms: P-SV, spherically symmetric case. Geophys Res Lett 21: $1663-1666$.

Furumura T, Kennett B L N and Furumura M (1998). Seismic wavefield calculation for laterally heterogeneous whole Earth models using the pseudospectral method. Geophys J Int 135: 845-860.

Herrmann R B (1979). SH-wave generation by dislocation source - a numerical study. Bull Seis Soc Amer 69: 1-15.

Igel $\mathrm{H}$ and Gudmundsson O (1997). Frequency-dependent effects on travel times and waveforms of long-period S and SS waves. Phys Earth Planet Inter 104: 229-246.

Igel H and Weber M (1995). SH-wave propagation in the whole mantle using high-order finite differences. Geophys Res Lett 22: 731-734.

Igel H and Weber M (1996). P-SV wave propagation in the Earth's mantle using finite differences: application to heterogeneous lowermost mantle structure. Geophys Res Lett 23: 415-418.

Jahnke G, Thorne M S, Cochard A and Igel H (2008). Global SH-wave propagation using a parallel axisymmetric spherical finite-difference scheme: application to whole mantle scattering. Geophys $J$ Int 173: 815-826, doi: 10.1111/j.1365-246X.2008.03744.x. 
Komatitsch D and Tromp J (2002). Spectral-element simulations of global seismic wave propagation - I. Validation. Geophys J Int 149: 390-412.

Nissen-Meyer T, Fournier A and Dahlen F A (2008). A 2-D spectral-element method for computing spherical-earth seismograms - II. Waves in solidfluid media. Geophys J Int 174: 873-888, doi:10.1111/j.1365-246X.2008. 03813.x.

Özdenvar T and McMechan G A (1996). Causes and reduction of numerical artefacts in pseudo-spectral wavefield extrapolation. Geophys $J$ Int 126: 819-828.

Toyokuni G, Takenaka H, Wang Y and Kennett B L N (2005). Quasispherical approach for seismic wave modeling in a 2-D slice of a global earth model with lateral heterogeneity. Geophys Res Lett 32: L09305, doi:10.1029/ 2004 6L022180.
Wang Y B and Takenaka H (2001). A multidomain approach of the Fourier pseudospectral method using discontinuous grid for elastic wave modeling. Earth Planets Space 53: 149-158.

Wang Y B, Takenaka H and Furumura T (2001). Modelling seismic wave propagation in a two-dimensional cylindrical whole-earth model using the pseudospectral method. Geophys J Int 145: 689-708.

Yan Z Z, Zhang H, Yang C C and Shi Y L (2009). Spectral element analysis on the characteristics of seismic wave propagation triggered by Wenchuan $M_{\mathrm{S}} 8.0$ earthquake. Science in China (Series D) 52: 764-773.

Zhao Z X, Xu J R and Horiuchi S (2001). Differentiation operation in the wave equation for the pseudospectral method with a staggered mesh. Earth Planets Space 53: 327-332. 